\title{
Loss of consciousness and convulsion induced by a ventricular tachycardia mimicking epilepsy in a patient with noncompaction cardiomyopathy: a case report
}

\author{
S. A. W. G. Dello • C. Kievit • P. H. Dunselman • \\ M. Alings
}

Published online: 5 July 2013

(C) The Author(s) 2013. This article is published with open access at Springerlink.com

\begin{abstract}
Convulsions and loss of consciousness can be caused by, among other things, arrhythmias, conduction disorders or epilepsy. In clinical practice it can be difficult to distinguish between these causes of syncope, even for well-trained specialists. Patients with cardiac syncope have a substantial risk of subsequent sudden death. We present a patient with previously unknown noncompaction cardiomyopathy in whom syncope induced by ventricular tachycardia was misinterpreted as epilepsy. We present this case report in order to underline the necessity for cardiological assessment in patients with assumed mild epilepsy or syncope of unknown origin.
\end{abstract}

Keywords Syncope · Epilepsy · Ventricular tachycardia · Noncompaction cardiomyopathy

\section{Introduction}

Convulsions and loss of consciousness in patients can be caused by arrhythmias, conduction disorders [1] or epilepsy [2]. When syncope is misinterpreted as epilepsy, this may not only lead to the unnecessary use of anticonvulsant drugs but, more importantly, life-threatening arrhythmias [3] can be

Simon A. W. G. Dello and Chris Kievit shared first authors.

S. A. W. G. Dello $\cdot$ C. Kievit • P. H. Dunselman · M. Alings $(\bowtie)$ Department of Cardiology, Amphia Hospital Breda, Molengracht 21, 4818 CK Breda, the Netherlands

e-mail: malings@amphia.nl missed which may lead to sudden unexpected death [4]. The aim of this case report is to present a patient with previously unknown noncompaction cardiomyopathy (NCCM) in whom syncope induced by ventricular tachycardia was misinterpreted as epilepsy, in order to underline the necessity for cardiological assessment in patients with assumed mild epilepsy or syncope of unknown origin.

\section{Case report}

A 57-year-old man without any history of cardiovascular disease was initially referred to the neurologist for analysis of collapse. An electroencephalogram (EEG) revealed an evident paroxysmal disturbance of function in the right frontotemporal lobe. He was diagnosed with mild focal epilepsy and was treated with levetiracetam without any result. A second EEG showed no EEG abnormalities; however, a simultaneous electrocardiogram (ECG) showed a sudden onset of a wide complex tachycardia when the patient collapsed (Fig. 1).

One year before admission the patient started to suffer from dizziness and collapse several times a day. He did not complain of palpitations or chest pain. In the last 2 months ordinary physical activity resulted in fatigue and dyspnoea (NYHA class II). Besides smoking 10 cigarettes a day, there were no other risk factors for cardiovascular disease. There was no family history of cardiovascular disease or sudden death. ECG showed a sinus rhythm with normal conduction times and no ST-T deviations. His blood pressure was 140/80 mmHg. Transthoracic echocardiography demonstrated 


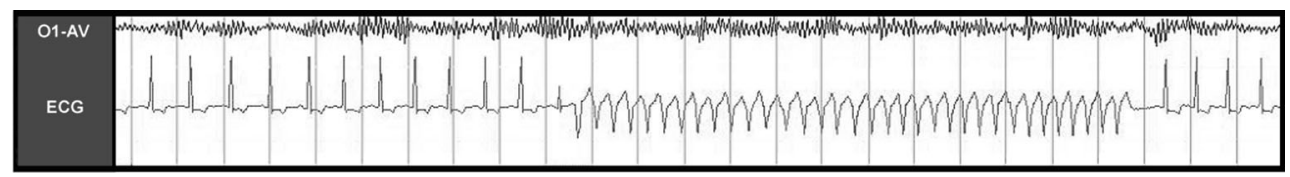

Fig. 1 A fragment of a 24-h EEG which shows a sudden onset of a wide complex tachycardia on a simultaneously recorded ECG, while there is no electrical disturbance in the EEG leads (only one of the EEG is shown, O1-AV)

an impaired systolic left ventricular function with an ejection fraction of $45 \%$. Apical segments were excessively thickened with prominent hypertrabeculation. The echocardiographic findings met the diagnostic criteria of a noncompaction cardiomyopathy (NCCM) [5]. Magnetic resonance imaging (MRI) of the heart confirmed the diagnosis of NCCM (Fig. 2). Telemetry monitoring showed monomorphic non-sustained ventricular tachycardias (nsVT) with a right bundle branch block configuration, a superior axis in the frontal plane and a frequency of 150-170 beats/min (Fig. 3). During these VTs the patient noticed dizziness without loss of consciousness. Coronary angiography demonstrated patent coronary arteries without significant stenosis.

The patient was treated with a beta-blocker and an ACEinhibitor which reduced the number and duration of nonsustained VTs and the patient became asymptomatic. Considering the NCCM with poor systolic LV function and symptomatic VTs without a treatable arrhythmogenic substrate, there was a class I (evidence level A) indication for ICD implantation according to the guidelines for the management of patients with ventricular arrhythmia and the prevention of sudden cardiac death (SCD) [6]. After

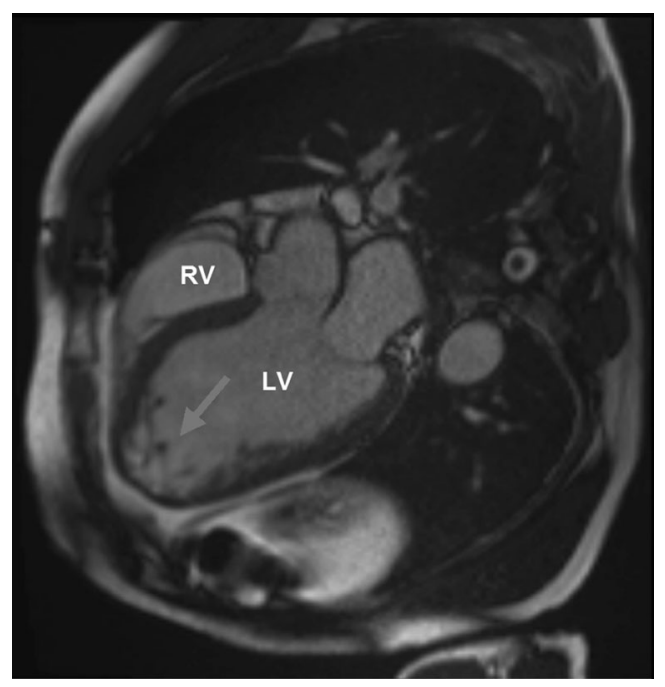

Fig. 2 MRI of the heart confirmed the diagnosis of noncompaction cardiomyopathy, excessive trabeculation (arrow) LV = left ventricle, $\mathrm{RV}=$ right ventricle uncomplicated ICD implantation the patient left our hospital in a good condition.

\section{Discussion}

Transient loss of consciousness (T-LOC) is defined as an abrupt, self-limiting complete loss of consciousness [1]. Cardiac syncope is caused by reduced global cerebral perfusion due to a sudden decrease in cardiac output. Arrhythmia and structural heart disease such as aortic stenosis and cardiomyopathy are the most important cardiac causes of syncope. Recognising cardiac syncope is of utmost importance because of all patients with T-LOC, the ones with cardiac syncope have the highest likelihood of subsequent sudden death [7]. Kapoor et al. [8] showed in 1983 that in the 12 months following presentation with cardiac syncope, sudden death was found to be six times more common than in patients with noncardiac syncope. It is unknown how often cardiac syncope is misdiagnosed as epilepsy.

The patient of this case report suffered from syncope induced by a ventricular tachycardia as a consequence of noncompaction cardiomyopathy (NCCM). NCCM is a relatively new and frequently overlooked diagnosis $[9,10]$. Even with the help of modern imaging modalities, such as contrast echocardiography and MRI, diagnosing NCCM can be difficult [10]. The clinical spectrum of NCCM shows a highly variable clinical presentation, ranging from asymptomatic to severely symptomatic with end-stage heart failure, lethal arrhythmias, and/or thromboembolic events [11]. Most cases are inherited, with a dominant inheritance pattern. There is a high prevalence of, mostly asymptomatic, cardiac disease (26\%) among first- and second-degree family members of patients with NCCM [12]. This warrants genetic counselling, DNA diagnostics, and cardiological family screening and offers an opportunity for early intervention $[12,13]$. There is no consensus on the treatment of NCCM. Caliskan et al. [14] showed in a relatively large group of NCCM patients that ICD therapy for primary or secondary prevention of SCD was appropriate in both groups supporting the application of ICD in these patients. However, although ICD seems to be an effective option for preventing $\mathrm{SCD}$, data on the long-term follow-up of NCCM patients remain scarce. 
Fig. 3 Telemetric rhythm observation: non-sustained VT, monomorphic, 170 complexes/ $\mathrm{min}$. The VT originated from the infero-apical left ventricle

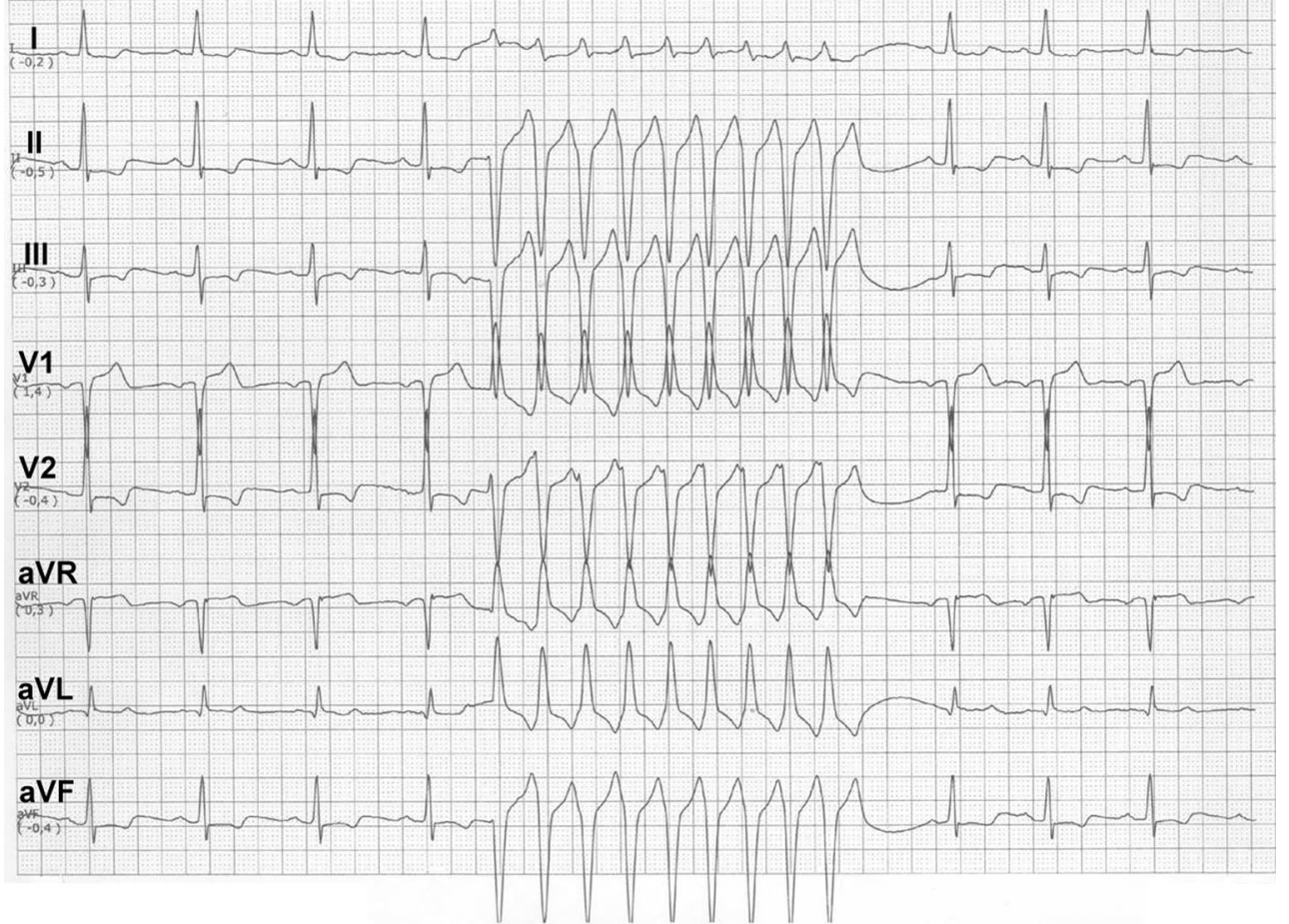

\section{Conclusion}

A substantial number of the patients with T-LOC have cardiac syncope and these patients have a substantial risk of subsequent sudden death. Sudden death may be preventable in these patients, which underlines the necessity for a cardiological assessment in patients with syncope.

Acknowledgments The authors would like to thank Dr. M.F.A.M. Sturm from the Department of Radiology, Amphia Hospital Breda, Breda, the Netherlands, for kindly providing access to the Cardiac Magnetic Resonance images.

Funding None.

\section{Conflict of interests None declared.}

Open Access This article is distributed under the terms of the Creative Commons Attribution License which permits any use, distribution, and reproduction in any medium, provided the original author(s) and the source are credited.

\section{References}

1. Moya A, Sutton R, Ammirati F, et al. Guidelines for the diagnosis and management of syncope (version 2009). Eur Heart J. 2009;30(21):2631-71.

2. Brophy GM, Bell R, Claassen J, et al. Guidelines for the evaluation and management of status epilepticus. Neurocrit Care. 2012;17(1):3-23.

3. van der Werf C, Postema PG. A young man with near-syncope. Neth Heart J. 2011;19(7-8):361-3.
4. Rodrigues Tda R, Sternick EB, Moreira MC. Epilepsy or syncope? An analysis of 55 consecutive patients with loss of consciousness, convulsions, falls, and no EEG abnormalities. Pacing Clin Electrophysiol. 2009;33(7):804-13.

5. Jenni R, Oechslin E, Schneider J, et al. Echocardiographic and pathoanatomical characteristics of isolated left ventricular noncompaction: a step towards classification as a distinct cardiomyopathy. Heart. 2001;86(6):666-71.

6. Zipes DP, Camm AJ, Borggrefe M, et al. Guidelines for management of patients with ventricular arrhythmias and the prevention of sudden cardiac death. Executive summary. Rev Esp Cardiol. 2006;59(12):1328.

7. Anderson J, O'Callaghan P. Cardiac syncope. Epilepsia. 2012;53 Suppl 7:34-41.

8. Kapoor WN, Karpf M, Wieand S, et al. A prospective evaluation and follow-up of patients with syncope. N Eng J Med. 1983;309(4):197-204.

9. Engberding R, Bender F. Identification of a rare congenital anomaly of the myocardium by two-dimensional echocardiography: persistence of isolated myocardial sinusoids. Am J Cardiol. 1984;53(11):1733-4.

10. Caliskan K. Noncompaction cardiomyopathy, a frequently overlooked entity (...but beware of over diagnosis!). Neth Heart J. 2012;20(10):387-8.

11. Weiford BC, Subbarao VD, Mulhern KM. Noncompaction of the ventricular myocardium. Circulation. 2004;109(24):2965-71.

12. Caliskan K, Michels M, Geleijnse ML, et al. Frequency of asymptomatic disease among family members with noncompaction cardiomyopathy. Am J Cardiol. 2012;110(10):1512-7.

13. Hoedemaekers YM, Caliskan K, Michels M, et al. The importance of genetic counseling, DNA diagnostics, and cardiologic family screening in left ventricular noncompaction cardiomyopathy. Circ Cardiovasc Genet. 2010;3(3):232-9.

14. Caliskan K, Szili-Torok T, Theuns DA, et al. Indications and outcome of implantable cardioverter-defibrillators for primary and secondary prophylaxis in patients with noncompaction cardiomyopathy. J Cardiovasc Electrophysiol. 2011;22(8):898-904. 